\title{
Avaliação da média de ressangramento em pacientes pediátricos com trombose da veia porta que fizeram uso de profilaxia endoscópica e/ou medicamentosa
}

\author{
Evaluation of the re bleeding average in pediatric patients with portal vein \\ thrombosis that used endoscopic and/or prophylactic drug therapy
}

\author{
Lígia Patrícia de Carvalho Batista Éboli ${ }^{1}$, Maria Carolina Amando do Nascimento \\ Matias' $^{2}$, Raquel Nogueira Cordeiro², Gabriela Maria Carvalho Araújoº \\ Emanuel Alexandre Amando Souza ${ }^{2}$, Diego Laurentino Lima ${ }^{3}$
}

Éboli LPCB, Matias MCAN, Cordeiro RN, Araújo GMC, Souza EAA, Lima DL. Avaliação da média de ressangramento em pacientes pediátricos com trombose da veia porta que fizeram uso de profilaxia endoscópica e/ou medicamentosa / Evaluation of the re bleeding average in pediatric patients with portal vein thrombosis that used endoscopic and/or prophylactic drug therapy. Rev Med (São Paulo). 2018 jul.-ago.;97(4):396-401.

RESUMO: Introdução: A hipertensão portal (HP) resulta da obstrução venosa portal extra-hepática cuja principal etiologia na faixa etária pediátrica é a trombose da veia porta. A complicação mais temida da HP é a hemorragia digestiva alta decorrente da ruptura das varizes esofágicas. Estima-se que cerca de 70-79\% das crianças com Trombose de veia prota (TVP) apresentarão pelo menos um episódio de hemorragia digestiva alta durante sua vida. A abordagem é dividida da seguinte forma: profilaxia primária, tratamento de episódios hemorrágicos agudos e profilaxia secundária. Os métodos profiláticos disponíveis englobam modalidades farmacológica, terapia endoscópica e cirurgia. Objetivos: avaliar a taxa de ressangramento por ruptura de varizes esofágicas e/ou gástricas em pacientes que utilizam profilaxia secundária com propranolol e/ou procedimentos endoscópicos, acompanhados no ambulatório de hepatologia pediátrica do IMIP. Método: Estudo analítico, retrospectivo, realizado por meio da análise do prontuário do paciente. $\mathrm{O}$ programa utilizado para análise estatística foi o software "R" versão 3.3.1 Resultados: A taxa global de ressangramento independente do tipo de profilaxia utilizada foi de 1,5 vezes. Os procedimentos mais utilizados para controle de sangramento foram o tratamento endoscópico com escleroterapia e ligadura elástica $(n=10)$, seguidos do uso de tratamento endoscópico e medicamentoso com propranolol $(n=9)$. Ao avaliar a frequência de ressangramento observa-se que os pacientes que realizaram apenas escleroterapia $(n=6)$ apresentaram média de ressangramento de 2,16 vezes. Os pacientes que realizaram apenas ligadura elástica $(n=2)$ ou propranolol $(\mathrm{n}=3)$ não apresentaram novos sangramentos. Conclusões: O presente estudo mostra uma menor média de ressangramento naqueles pacientes que fizeram uso de ligadura elástica ou propranolol isoladamente. No entanto, novos estudos na faixa etária pediátrica, são necessários para avaliação da real eficácia e segurança de cada método especificamente.

Descritores: Hipertensão portal; Varizes esofágicas e gástricas; Propranolol; Escleroterapia; Ligadura.

ABSTRACT: Introduction: Portal hypertension is a consequence of extra-hepatic portal vein obstruction, and portal vein thrombosis is its main etiology in pediatric age. The most feared portal hypertension complication is upper digestive hemorrhage caused by rupture of esophageal varicose veins. It's estimated that

1. Hepatologista pediatra no Instituto de Medicina Integral Prof. Fernando Figueira (IMIP), Unidade de Transplante de Fígado do Hospital Universitário Oswaldo Cruz. Gastroenterologista e hepatologista pediatra da clínica Real Hepato do Real Hospital Português, Tutora do curso de medicina da Faculdade Pernambucana de Saúde (FPS). ORCID: https://orcid.org/0000-0001-6821-6658. Email: lipcbs@gmail.com.

2. Acadêmicos de Medicina, Faculdade Pernambucana de Saúde (FPS), Recife, PE, Brasil. ORCID: Matias MCAN - https://orcid.org/0000-0003-19347701; Cordeiro RN - ORCID: https://orcid.org/0000-0002-0238-8374; Araújo GMC - ORCID: https://orcid.org/0000-0001-5655-7666; Souza EAA - ORCID: https://orcid.org/0000-0001-8503-1583.Email: carolina_amando@hotmail.com, raquelnogueiracordeiro@gmail.com, gabrielamaria11@ hotmail.com, emanuel1118@hotmail.com.

3. Residente de cirurgia geral do Hospital dos Servidores do Estado de Pernambuco, Recife, Brasil. ORCID https://orcid.org/0000-0003-1257-117X. Email: dilaurentino@gmail.com.

Autor Correspondente: Raquel Nogueira Cordeiro. Rua dos Coelhos, 300. Boa Vista. Recife, PE, Brasil. CEP: 52.050-300. Email: raquelnogueiracordeiro@ gmail.com. 
Éboli LPCB, et al. Avaliação da média de ressangramento em pacientes pediátricos com trombose.

over 70-79 per cent of children with deep vein thrombosis (DVT) will present at least one episode of upper digestive hemorrhage on their lives. The approach is divided in: primary prophylaxis, treatment of acute hemorrhage episode and secondary prophylaxis. Available prophylactic methods are: pharmacologic, endoscopic therapy, and surgery. Objectives: Evaluate the rebleeding rate due to esophageal and/or gastric varicose veins rupture in patients using secondary prophylaxis with propanolol and/or endoscopic procedures/methods, followed up in IMIP's pediatric hepatology ambulatorial service. Methods: It's an analytical, and retrospective study, with the analysis of patient's medical records. A computer software "R" version 3.3.1. was used for the statistical analysis. Results: Rebleeding global rate was 1,5 times, in spite of prophylaxis's type used. The most utilized procedures for

\section{INTRODUÇÃO}

Чipertensão portal (HP) é definida como aumento da pressão no sistema porta acima de $5 \mathrm{mmHg}^{1,2}$. A HP resulta, mais frequentemente, da obstrução venosa portal extra-hepática (OVPEH) $)^{3,4}$ apresentando como principal etiologia, em pediatria, a trombose da veia porta $(\mathrm{TVP})^{4,5}$. A complicação mais temida da HP é a hemorragia digestiva alta (HDA), decorrente da ruptura das varizes esofágicas ${ }^{3,6}$. O evento hemorrágico resultante da HP é causador de grande morbimortalidade, chegando a ter taxas de mortalidade de até $30 \%{ }^{2}$. Estima-se que cerca de 70-79\% das crianças com TVP apresentarão pelo menos um episódio de HDA durante sua vida ${ }^{4,5}$.

Crianças com trombose de veia porta também costumam apresentar esplenomegalia ao exame físico e manifestações de hiperesplenismo como trombocitopenia, anemia e leucopenia. Plaquetopenia e esplenomegalia parecem ser fatores preditivos independentes de varizes de grosso calibre?

Qualquer intervenção tomada para prevenir o primeiro episódio de sangramento consiste na profilaxia primária ${ }^{8,910}$. Já a profilaxia secundária se refere a prevenção de ressangramento e deve ser realizado em torno de 2 semanas após o primeiro episódio de sangramento $^{8,10,11,12}$.

O propranolol é um beta-bloqueador (BB) não seletivo que tem eficácia demonstrada tanto na prevenção primária quanto na secundária para profilaxia de HDA em adultos ${ }^{13}$. O seu mecanismo de ação se baseia na diminuição do débito cardíaco e indução à vasoconstricção esplâncnica por bloqueio de receptores $\beta-1$ e $\beta-2$, reduzindo a pressão porta em mais de $20 \%$ e prevenindo o desenvolvimento e a ruptura das varizes ${ }^{2,14}$. Os estudos sobre a terapia com propranolol na faixa etária pediátrica são escassos e por isso o seu uso tem sido empírico ${ }^{5,13,15,16}$. Além disso, há receio do seu uso pelo risco teórico de inibir uma das principais vias de compensação em caso de choque hipovolêmico em crianças com $\mathrm{HDA}^{4}$.

A escleroterapia endoscópica das varizes (EEV) é usada de forma complementar com a ligadura endoscópica bleeding control were endoscopic treatment with sclerotherapy and elastic ligature $(\mathrm{N}=10)$ followed by endoscopic and drug therapy with propanolol ( $\mathrm{N}=9$ ). Analyzing rebleeding frequency, it can be noticed that patients which the only treatment realized was sclerotherapy presented re-bleed average of 2,16 times. Patients who realized only elastic ligature $(\mathrm{N}=2)$ or propanolol $(\mathrm{N}=3)$ had not presented new bleeding episodes. Conclusions: The present study reveals a small average of rebleeding in patients that used only elastic ligature or propanolol. However, new studies in pediatric age group are needed to evaluate the security and efficacy of specific methods.

Keywords: Hypertension, portal; Esophageal gastric varices; Propanolol; Sclerotherapy; Ligation.

ou então em casos tecnicamente difíceis de realizar apenas a ligadura ${ }^{11,17,18}$. As complicações da EEV incluem febre, desconforto/dor retroesternal, disfagia, hemorragia induzida por injeção, ulceração esofágica com hemorragia tardia, estenoses esofágicas, perfuração esofágica, mediastinite, derrame pleural, fístula broncoesofágica, síndrome de dificuldade respiratória aguda e infecção ${ }^{18}$.

Ligadura endoscópica (LE) das varizes consiste em um procedimento realizado a partir da introdução do endoscópio até a junção esôfago-gástrica, sucção da variz pelo aparelho e colocação de um anel elástico em sua base, provocando isquemia da mesma ${ }^{19}$. É um método preferível no controle de sangramento agudo e mais seguro quando comparado à escleroterapia para profilaxia secundária ${ }^{20,21,22}$. No entanto, escleroterapia não pode ser totalmente descartada, especialmente em crianças, pois continua sendo a única opção terapêutica em menores de 2 anos de idade ${ }^{23}$. Um importante efeito adverso relacionado ao próprio procedimento de ligadura elástica é a formação de úlceras que pode conduzir a um defeito grave na parede gástrica ${ }^{24}$.

Derivação portossistêmica cirúrgica descomprime diretamente o sistema venoso portal e restaura o fluxo sanguíneo portal para o fígado em pacientes com $\mathrm{TVP}^{4}$. O mesorex (shunt seletivo no qual se liga a veia mesentérica superior ao ramo intra-hepático da veia porta através de um enxerto da jugular) é hoje o procedimento mais indicado como profilaxia primária em crianças com $\mathrm{TVP}^{19}$. No entanto, a escassez de profissionais para realizar esse procedimento gera a necessidade de novas formas de tratamento mais acessíveis.

Logo, avaliar a taxa de ressangramento em pacientes pediátricos com trombose de veia porta e varizes esofágicas que fazem ou fizeram uso de algum método profilático para controle de sangramento e erradicação de varizes esofágicas pode esclarecer qual desses métodos está relacionado a menor taxa de ressangramento.

\section{MÉTODO}

Foi realizado um estudo analítico, retrospectivo. 
A população de estudo foi composta por crianças e adolescentes com trombose da veia porta atendidos no ambulatório de hepatologia pediátrica do Instituto de Medicina Integral Fernando Figueira (IMIP).

Do total de 57 pacientes com diagnóstico de trombose da veia porta atendidos no ambulatório de hepatologia pediátrica do Instituto de Medicina Integral Fernando Figueira (IMIP), 42 participaram da pesquisa. Os demais não participaram da pesquisa devido à perda do seguimento ou por não possuírem os dados necessários em seus prontuários.

A coleta de dados ocorreu durante os meses de junho de 2016 a fevereiro de 2017, período em que foram analisados os prontuários dos pacientes coletando informações sobre idade, sexo, taxa de ressangramento por ruptura de varizes esofágicas, número de plaquetas, uso ou não de propranolol, realização de ligadura elástica ou escleroterapia para controle de sangramento e erradicação de varizes esofágicas.

O Termo de Consentimento Livre e Esclarecido (TCLE) foi coletado no momento da consulta ambulatorial, sendo necessária a leitura e concordância com os termos do estudo para ter acesso ao prontuário do paciente.

Ao final da coleta, os dados foram compilados em tabela de Excel e foram analisados estatisticamente pelo software "R" versão 3.3.1. Para comparar as médias entre variáveis independentes foi utilizado o teste $\mathrm{T}$ de student e o teste $\mathrm{F}$ quando se tinha mais de duas categorias na variável categórica. Para avaliar o grau de relação linear entre duas variáveis quantitativas foi utilizado o coeficiente de correlação de Pearson. Considerou-se resultado estatisticamente significante o valor de $p$ menor que 0,05 .

O presente trabalho foi aprovado pelo Comitê de Ética e Pesquisa em Seres Humanos do Instituto de Medicina Integral Fernando Figueira (IMIP), sob CAE de número 54703916.1.0000.5201.

\section{RESULTADOS}

Um total de 42 pacientes foram incluídos na pesquisa. Destes, 27 (64,2\%) eram do sexo masculino. A média de idade foi de 10,6 anos ( $D P=4,04 ; 4-18$ anos).

A taxa global de ressangramento independente do tipo de profilaxia utilizada foi de 1,5 vezes $(D P=1,6 ; 0-7$ vezes). Avaliando a taxa de ressangramento com relação ao gênero, não houve diferença estatisticamente significante $(p=0,36)$ entre masculino (média 1,$3 ; D P=1,3)$ e feminino (média 1,$8 ; D P=1,95$ ).

Dos 42 pacientes analisados, 37 (88\%), apresentavam plaquetopenia (plaquetas $<150$ mil) sendo a média do número de plaquetas de $93.6 \mathrm{mil}(D P=57,5 \mathrm{mil} ; 21 \mathrm{mil} \mathrm{-}$ 312 mil).
Tabela 1. Análise descritivas das variáveis frequência de sangramento, idade e número de plaquetas

\begin{tabular}{lcccc}
\hline Variáveis & Média & $\begin{array}{c}\text { Desvio } \\
\text { padrão }\end{array}$ & Mínimo & Máximo \\
$\begin{array}{l}\text { Frequência de } \\
\text { ressangramento }\end{array}$ & 1,5 & 1,61 & 0 & 7 \\
\hline Idade & 10,6 & 4,04 & 4 & 18 \\
\hline Plaquetas & 93694 & 57508 & 21000 & 312000 \\
\hline
\end{tabular}

A correlação encontrada entre número de plaquetas e frequência de ressangramento foi fraca e não significativa $(\mathrm{r}=-0,28$ e $\mathrm{p}=0,24)$

Comparando-se a taxa de ressangramento com os métodos profiláticos utilizados, houve uma diferença estatística entre os resultados $(\mathrm{p}=0,04)$. Os procedimentos mais utilizados para controle de sangramento foram o tratamento endoscópico com escleroterapia e ligadura elástica $(n=10)$, seguidos do uso de tratamento endoscópico e medicamentoso com propranolol $(n=9)$. Ao avaliar a frequência de ressangramento observa-se que os pacientes que realizaram apenas escleroterapia $(n=6)$ apresentaram média de ressangramento de 2,16 vezes $(\mathrm{DP}=1,32)$. Os pacientes que realizaram apenas ligadura elástica $(n=2)$ ou propranolol $(n=3)$ não apresentaram novos sangramentos.

Tabela 2. Associação entre método de profilaxia secundária utilizado e a média de ressangramento por ruptura de varizes esofágicas

\begin{tabular}{lcccc}
\hline \multicolumn{4}{c}{ Hemorragia digestiva alta } & \\
\hline & $\begin{array}{c}\text { Média de } \\
\text { sangramento }\end{array}$ & $\begin{array}{c}\text { Número de } \\
\text { pacientes }\end{array}$ & $\begin{array}{c}\text { Desvio } \\
\text { padrão }\end{array}$ & p \\
\hline Nenhum & 0,16 & 6 & 0,4 \\
\hline Escleroterapia & 2,16 & 6 & 1,32 \\
\hline $\begin{array}{l}\text { Ligadura elástica } \\
\text { Propranolol }\end{array}$ & 0 & 2 & 0 \\
\hline $\begin{array}{l}\text { Escleroterapia }+ \\
\text { Ligadura elástica }\end{array}$ & 2,3 & 10 & 2 \\
\hline $\begin{array}{l}\text { Escleroterapia }+ \\
\text { propranolol }\end{array}$ & 1 & 3 & 1 \\
\hline $\begin{array}{l}\text { Ligadura elástica }+ \\
\text { propranolol } \\
\text { Escleroterapia }+\end{array}$ & 2,3 & 3 & 1,52 \\
$\begin{array}{l}\text { Ligadura elástica }+ \\
\text { propranolol }\end{array}$ & 1,8 & 9 & 1,53 \\
\hline
\end{tabular}


Éboli LPCB, et al. Avaliação da média de ressangramento em pacientes pediátricos com trombose.

\section{DISCUSSÃO}

Estima-se que e após o controle do primeiro evento o risco de ressangramento em pacientes com trombose de veia porta e varizes esofágicas é de aproximadamente $70 \%{ }^{25,26}$. A ruptura das varizes esofágicas está relacionada a uma elevada taxa de morbimortalidade, afetando também os familiares que tornam-se ansiosos em relação a imprevisibilidade de outro sangramento. A abordagem é dividida da seguinte forma: profilaxia primária para prevenir um episódio inicial de hemorragia varicosa, tratamento de episódios hemorrágicos agudos e profilaxia secundária para prevenir hemorragias recorrentes ${ }^{27}$. Os métodos profiláticos disponíveis englobam modalidades farmacológica, terapia endoscópica e cirúrgica. Em Pernambuco não há profissionais para realizar a derivação portossistêmica cirúrgica, embora seja a profilaxia mais indicada. Entre os participantes do nosso estudo, a maioria faz uso de algum método profilático, apenas 6 pacientes $(14,28 \%)$ que não tinham utilizado nenhuma medida até o momento do estudo.

$\mathrm{O}$ uso de beta-bloqueador (BB) não seletivo está bem estabelecido em adultos ${ }^{20}$, porém ainda há poucos estudos que avaliam a sua segurança e eficácia em crianças e adolescentes. Em uma análise que comparou o BB com o placebo foi evidenciado uma redução do risco de hemorragia de varizes de 14 a $30 \%{ }^{28}$ além de ter sido associada a uma redução na mortalidade ${ }^{29}$. A porcentagem de pacientes que fazem uso do propranolol na nossa pesquisa foi de $42,8 \%$, e, destes, apenas $7,1 \%$ $(n=3)$ faziam uso isolado e tiveram uma média 0 de sangramento, sugerindo uma boa eficácia para prevenção de um evento hemorrágico. A dose mínima e máxima utilizadas nesses 3 pacientes foram de $20 \mathrm{mg} /$ dia e 40 $\mathrm{mg}$ /dia. Os outros $35,7 \%$ utilizaram o BB associado com ligadura elástica e/ou escleroterapia.

Em nossa observação, a monoterapia com BB versus a monoterapia com ligadura elástica se mostraram equivalentes, com uma taxa de 0 sangramento, resultado que está de acordo com o consenso entre os especialistas ${ }^{18}$. Dados que vão de encontro ao nosso estudo, foram encontrados em uma metanálise que incluiu 12 estudos comparando os métodos profiláticos utilizados por 839 indivíduos adultos (410 com ligadura elástica e 429 com $\beta$-bloqueador) demonstrando a superioridade da ligadura elástica ${ }^{30}$. Em outro estudo que comparou a terapia endoscópica através da ligadura elástica versus propranolol em crianças e adolescentes, o betabloqueador também apresentou piores resultados no ressurgimento de varizes esofágicas (60\% vs $44 \%)$ e na taxa de ressangramento (40\% vs $27 \%$ ), porém devido a amostra pequena não foi possível obter conclusões ${ }^{15}$.

A escleroterapia foi utilizada como terapia única em 6 pacientes $(14,2 \%)$, e teve uma média de
2 sangramentos, o mínimo foi de 1 e o máximo de 4 sangramentos. Analisando o uso isolado de ligadura elástica e da escleroterapia, os pacientes que utilizaram apenas ligadura elástica não apresentaram novos sangramentos, enquanto a média de ressangramento naqueles que fizeram apenas escleroterapia foi de 2,16 vezes. Zargar et al. ${ }^{22}$ avaliando 49 pacientes ( 25 realizaram LE e 24 fizeram escleroterapia) encontraram vantagens significativas do grupo da LE em relação à escleroterapia na prevenção da hemorragia digestiva alta (4\% vs 25\%). O motivo mais provável para a menor taxa de HDA no grupo da LE é a sua capacidade de erradicar as varizes esofágicas em poucas sessões. A erradicação das varizes esofágicas pela LE é alcançada em aproximadamente $90 \%$ dos pacientes ${ }^{31}$, embora a recorrência não seja rara.

De nove ensaios controlados randomizados que compararam a escleroterapia a longo prazo com tratamento farmacológico usando b-bloqueadores ${ }^{32-40}$, três mostraram que a escleroterapia era superior ao $\mathrm{BB}^{34,38,40}$, cinco estudos não mostraram diferença entre eles e apenas um mostrou superioridade do propranolol. Os dados do nosso estudo mostram uma taxa de ressangramento menor nos pacientes que fizeram uso de BB quando comparado com escleroterapia (0 versus 2,16).

A associação do $\beta$-bloqueador com métodos endoscópicos mostrou uma média de 2,3 sangramentos em pacientes que usaram propranol e ligadura elástica, enquanto aqueles que utilizaram escleroterapia e propranolol tiveram uma média de 1 ressangramento. Um estudo de Sarin et al. mostrou que ligadura endoscópica mais bloqueador $\beta$-adrenérgico parece não oferecer nenhum benefício em termos de prevenção do sangramento quando comparado ao endoscópico ligadura de banda sozinha ${ }^{41}$, o que corrobora com os achados do presente estudo.

$\mathrm{O}$ número reduzido de pacientes que fizeram uso de determinada profilaxia isoladamente além do caráter retrospectivo podem ser considerados limitações do presente estudos. O fato de não terem publicações na faixa etária pediátrica sobre o assunto, principalmente de caráter prospectivo para avaliar eficácia, dificulta ainda mais na tomada de decisão do especialista em escolhar determinado método profilático de ressangramento nos pacientes com hipertensão portal secundária a trombose de veia porta.

Este estudo abre o caminho para que novos sejam realizados no intuito de avaliar eficácia e segurança dos métodos disponíveis para profilaxia secundária de hemorragia digestiva alta por ruptura de varizes esofágicas em crianças.

\section{CONCLUSÃO}

O manejo para a prevenção da hemorragia na 
Éboli LPCB, et al. Avaliação da média de ressangramento em pacientes pediátricos com trombose.

hipertensão portal é complexo na faixa etária pediátrica e ainda não está bem definido. O presente estudo mostrou uma menor média de ressangramento naqueles pacientes que fizeram uso de ligadura elástica ou propranolol isoladamente. No entanto, novos estudos são necessários para avaliação da real eficácia e segurança de cada método especificamente.

Participação dos autores: Informamos para devido fins que o artigo foi confeccionado em conjunto pelo grupo de autores com o grau de participação seguinte: Coleta de dados (Gabriela Maria Carvalho Araújo, Emanuel Alexandre Amando Souza e Maria Carolina Amando do Nascimento Matias Acadêmicos de Medicina). Orientação organizacional e sobre a essência, argumentação e relevância do trabalho: (Dra. Lígia Éboli - Médica Pediatra Hepatologista). Análise, pesquisa dos artigos, leitura e exclusão de pesquisas não pertinentes ao envolvimento do tema escolhido: (Raquel Nogueira Cordeiro - Acadêmica de Medicina e Dr. Diego Laurentino Lima - Residente de Cirurgia Geral). Leitura e escrita do conteúdo: (Maria Carolina Amando do Nascimento Matias, Dra. Lígia Éboli, Raquel Nogueira Cordeiro Diego e Diego Laurentino Lima). Revisão do texto quanto a integridade e veracidade quanto as fontes utilizadas: (Dra. Lígia Éboli e Dr. Diego Laurentino Lima). Dessa forma, o grupo de autores certifica participação conjunta na confecção do artigo, esperando contribuir no tema em questão, com uma síntese sobre o assunto em pontos atuais de conduta e definições baseado em trabalhos anteriores validados e publicados em revistas reconhecidas.

\section{REFERÊNCIAS}

1. Tiani C. Abraldes JG, Bosch J. Portal hypertension: preprimary and primary prophylaxis of variceal bleeding. Dig Liver Dis. 2008;40(5):318-27. doi: 10.1016/j. dld.2007.12.004.

2. Mileti E, Rosenthal P. Management of portal hypertension in children. Curr Gastroenterol Rep. 2011;13(1):10-6. doi: 10.1007/s11894-010-0151-y.

3. Wani AH, Shah OJ, Zargar SA. Management of variceal hemorrhage in children with extrahepatic portal venous obstruction-shunt surgery versus endoscopic sclerotherapy. Indian J Surg. 2011c;73(6):409-13. doi: 10.1007/s12262011-0345-z.

4. Giouleme O, Theocharidou E. Management of portal hypertension in children with portal vein thrombosis. J Pediatr Gastroenterol Nutr. 2013;57(4):419-25. doi: 10.1097/MPG.0b013e3182a1cd7f.

5. Ferri P M, Ferreira AR, Fagundes EDT, Liu SM, Roquete MLV, Penna FJ. Portal vein thrombosis in children and adolescents: 20 years experience of a pediatric hepatology reference center. Arq Gastroenterol. 2012;49(1):69-76. doi: 10.1590/S0004-28032012000100012.

6. Kim SK, Lee KA, Sauk S, Korenblat K. Comparison of transjugular intrahepatic portosystemic shunt with covered stent and ballon-occluded retrograde transvenous obliteration in managing isolated gastric varices. Korean J Radiol. 2017;18(2):345-54. doi: 10.3348/kjr.2017.18.2.345.

7. Fagundes ED, Ferreira AR, Roquete ML, Penna FJ, Goulart EM, Figueiredo Filho PP, et al. Clinical and laboratory predictors of esophageal varices in children and adolescents with portal hypertension syndrome. J Pediatr Gastroenterol Nutr. 2008;46(2):178-83. doi: 10.1097/ MPG.0b013e318156ff07.

8. Coelho FF, Perini MV, Kruger JAP, Fonseca GM, Araújo RLC de, Makdissi FF, et al . Management of variceal hemorrhage: current concepts. ABCD Arq Bras Cir Dig. 2014;27(2):138-44. doi: 10.1590/S010267202014000200011 .

9. Bittencourt PL, Farias AQ, Strauss E, Mattos AA. Variceal bleeding: consensus meeting report from the Brazilian Society of Hepatology. Arq Gastroenterol. 2010;47(2):20216. doi: 10.1590/S0004-28032010000200017.
10. Biecker E. Portal hypertension and gastrointestinal bleeding: diagnosis, prevention and management. World J Gastroenterol. 2013;19(31):5035-50. doi: 10.3748/wjg. v19.i31.5035.

11. Pimenta JR, Ferreira AR, Fagundes EDT, Bittencourt PFS, Moura AM, Carvalho SD. Evaluation of endoscopic secondary prophylaxis in children and adolescents with esophageal varices. Arq Gastroenterol. 2017;54(1):21-6. http://dx.doi.org/10.1590/s0004-2803.2017v54n1-04.

12. Ling, SC. Portal hypertension in children. Clin Liver Dis. 2012;1:139-42. https://doi.org/10.1002/cld.79.

13. Sarin SK, Gupta N, Jha SK, Agrawal A, Mishra SR, Sharma $\mathrm{BC}$, et al. Equal efficacy of endoscopic variceal ligation and propranolol in preventing variceal bleeding in patients with noncirrhotic portal hypertension. Gastroenterology. 2010;139(4):1238-45. doi: 10.1053/j.gastro.2010.06.017.

14. Araujo EA, Torres FSC, Carneiro FS, Costa KV, Guiotti MTG, Lopes RD, et al. Profilaxia dos sangramentos gastrintestinais em Medicina de Urgência. Rev Bras Clin Med. 2010;8(4):333-7.

15. Santos JMR dos, Ferreira AR, Fagundes EDT, et al. Endoscopic and pharmacological secondary prophylaxis in children and adolescents with esophageal varices. J Pediatr Gastroenterol Nutr. 2013;56(1):93-8 doi: 10.1097/ MPG.0b013e318267c334.

16. Santambrogio R, Opocher E, Costa M, Bruno S, Ceretti AP, Spina GP. Natural history of a randomized trial comparing distal spleno-renal shunt with endoscopicsclerotherapy in the prevention of variceal rebleeding: A lesson from the past. World J Gastroenterol. 2006;12(39):6331-38. doi: 10.3748/ wjg.v12.i39.6331.

17. Costaguta A, Alvarez F. Etiology and Management of Hemorrhagic Complications of Portal Hypertension in Children. International Journal of Hepatology. 2012;(2012):879163. Doi: 10.1155/2012/879163

18. Hwang JH, Shergill AK, Acosta RD, Chandrasekhara V, Chathadi KV, Decker GA, et al. The role of endoscopy in the management of variceal hemorrhage. Gastrointest Endosc. 2014;80(2):221-7. doi: 10.1016/j.gie.2013.07.023.

19. Santos JMR, Ferreira AR, Fagundes EDT, Ferreira APS, Ferreira LS, Magalhães MCR, et al. Profilaxia secundária 
Éboli LPCB, et al. Avaliação da média de ressangramento em pacientes pediátricos com trombose.

endoscópica e medicamentosa em crianças e adolescentes com varizes esofágicas. Rev Med Minas Gerais. 2011;21(4 Supl 1):28-34.

20. Shneider BL, de Ville de Goyet J, Leung DH, Srivastava A, Ling SC, Duché M, et al. Primary prophylaxis of variceal bleeding in children and the role of MesoRex Bypass: summary of the Baveno VI Pediatric Satellite Symposium. Hepatology. 2016;63:1368-80. doi: 10.1002/hep.28153.

21. Costaguta A, Alvarez F. Etiology and management of hemorrhagic complications of portal hypertension in children. Int J Hepatol. 2012;2012:879163. doi: $10.1155 / 2012 / 879163$.

22. Zargar SA, Javid G, Khan BA, Yattoo GN, Shah AH, Gulzar GM, et al. Endoscopic ligation compared with sclerotherapy for bleeding esophageal varices in children with extrahepatic portal venous obstruction. Hepatology. 2012;36:666-72. doi: 10.1053/jhep.2002.35278

23. Poddar U, Bhatnagar S, Yachha S. Endoscopic band ligation followed by sclerotherapy: Is it superior to sclerotherapy in children with extrahepatic portal venous obstruction?. J Gastroenterol Hepatol. 2011;26(2):255-9. doi: $10.1111 / \mathrm{j} .1440-1746.2010 .06397 . x$

24. Hashizume M, Akahoshi T, Tomikawa M. Management of gastric varices. J Gastroenterol Hepatol. 2011;26:1102-8. doi: 10.1111/j.1440-1746.2010.06572.x.

25. Lo GH, Lai KH, Cheng JS, Chen MH, Huang HC, Hsu PI, et al. Endoscopic variceal ligation plus nadolol and sucralfate compared with ligation alone for the prevention of variceal rebleeding: a prospective, randomized trial. Hepatology. 2000;32:461-5. doi: 10.1053/jhep.2000.16236.

26. Helmy A, Hayes P. Review article: current endoscopic therapeutic options in the management of variceal bleeding. Alimentary Pharmacol Therap. 2001;15(5):575-94. https:// doi.org/10.1046/j.1365-2036.2001.00950.x.

27. Schettino GCM, Fagundes EDT, Roquete MLV, Ferreira AR, Penna FJ. Trombose de veia porta em crianças e adolescentes. J. Pediatr. 2006;82(3):171-8.

28. D'Amico G, Pagliaro L, Bosch J. Pharmacological treatment of portal hypertension: an evidence-based approach. Sem Liv Dis. 1999;19:475-505. doi: 10.1055/s-2007-1007133.

29. Chen W, Nikolova D, Frederiksen SL, Gluud C: Betablockers reduce mortality in cirrhotic patients with oesophageal varices who have never bled. J Hepatol. 2004;40(suppl 1):67. doi: 10.1016/s0168-8278(04)90208-5.

30. Garcia-Pagan JC, Bosch J. Endoscopic band ligation in the treatment of portal hypertension. Nat.Clin Pract. Gastroenterol Hepatol. 2005;2:526-35. doi: 10.1038/ ncpgasthep0323.

31. Miyaaki H, Ichikawa T, Taura N, Miuma S, Isomoto H, Nakao K. Endoscopic management of esophagogastric varices in Japan. Ann Transl Med. 2014;2(5):42. doi: 10.3978/j.issn.2305-5839.2014.05.02.

32. Fleig WE, Stange EF, Hunecke R, Schönborn W, Hurler U, Rainer K, et al. Prevention of recurrent bleeding in cirrhotics with recent variceal hemorrhage: Prospective, randomized comparison of propranolol and sclerotherapy. Hepatology. 1987;7:355-61. https://doi.org/10.1002/hep.1840070224.

33. Dollet JM, Champigneulle B, Patris A, Bigard MA, Gaucher P. Endoscopic sclerotherapy versus propranolol after hemorrhage caused by rupture of esophageal varices in patients with cirrhosis. Results of a 4-year randomized study. Gastroenterol Clin Biol. 1988;12(3):234-9.

34. Alexandrino PT, Martin Alves M, Pinto Correia J. Propranolol or endoscopic sclerotherapy in the prevention of recurrence of variceal bleeding. A prospective, randomized clinical trial. J Hepatol. 1988;7:175-85. doi: https://doi.org/10.1016/S0168-8278(88)80480-X.

35. Liu JD, Jeng YS, Chen PH. Endoscopic injection sclerotherapy and propranolol in the prevention of recurrent variceal bleeding. In: $7^{\text {th }}$ Gastroenterology World Congress. Abstract Book. 1990. p.1181

36. Westaby D, Polson RJ, Gimson AE, Hayes PC, Hayllar K, Williams R. A controlled trial of oral propranolol compared with injection sclerotherapy for the long-term management of variceal bleeding. Hepatology. 1990;11:353-9. https:// doi.org/10.1002/hep.1840110304.

37. Rossi V, Calès P, Burtin P, Charneau J, Person B, Pujo $P$, et al. Prevention of recurrent variceal bleeding in alcoholic cirrhotic patients: a prospective controlled trial of propranolol and sclerotherapy. J Hepatol. 1991;12:283-9. doi: https://doi.org/10.1016/0168-8278(91)90828-Y.

38. Martin T, Taupignon A, Lavignolle A, Perrin D, Le Bodic L. Prevention of recurrent hemorrhage in patients with cirrhosis. Results of a controlled trial of propranolol versus endoscopic sclerotherapy. Gastroenterol Clin Biol. 1991;15(11):833-7.

39. Dasarathy S, Dwivedi M, Bhargava DK, Sundaram $\mathrm{KR}$, Ramachandran K. A prospective randomized trial comparing repeated endoscopic sclerotherapy and propranolol in decompensated (child class B and C) cirrhotic patients. Hepatology. 1992;16:89-94. https://doi. org/10.1002/hep.1840160116.

40. Terés J, Bosch J, Bordas JM, Garcia Pagán JC, Feu F, Cirera I, et al. Propranolol vs sclerotherapy in the prevention of variceal rebleeding: a randomised controlled trial. Gastroenterology. 1993;105:1508-14.

41. Sarin SK, Wadhawan M, Agarwal SR, Tyagi P, Sharma BC. Endoscopic variceal ligation plus propranolol versus endoscopic variceal ligation alone in primary prophylaxis of variceal bleeding. Am J Gastroenterol. 2005;4:797-804 . doi: $10.1111 / j .1572-0241.2005 .40468 . x$ 